\title{
Disks and winds in Young Solar-Type Stars: the Magnetic Connection
}

\author{
C. Dougados ${ }^{1}$, J. Bouvier ${ }^{1}$, J. Ferreira ${ }^{1}$ and S. Cabrit ${ }^{2}$ \\ ${ }^{1}$ Laboratoire d'Astrophysique de Grenoble, BP53, 38041 Grenoble Cédex 9 France \\ 2 Observatoire de Paris, LERMA, 61 Avenue de l'Observatoire, 75014 Paris, France \\ email: Catherine.Dougados@obs.ujf-grenoble.fr
}

\begin{abstract}
I discuss in this contribution the accretion and ejection processes occurring in solartype young stars. Understanding these two important processes, and their link, is one of the major issues in star formation. The magnetic field is thought to play a central role in both extracting the angular momentum from the disk and directing the accretion flow onto the star. I will focus on the well studied $\mathrm{T}$ Tauri stars, optically-revealed pre-main sequence stars with ages 1-10 Myrs and mass $\simeq 0.5 \mathrm{M}_{\odot}$. In the first part of this contribution, I present the current paradigm for magnetically channeled accretion, where the stellar magnetic field truncates the disk and directs the accretion flow and discuss recent observations, which indicate that this process is non-axisymmetric and time-dependent. I then turn to the study of the supersonic collimated jets observed in young stars. Magneto-hydrodynamic processes are the most likely driving mechanism. I present the main steady and non-steady outflow models, as well as constraints brought by recent high-resolution studies. I finally discuss the origin of time variability in jets.
\end{abstract}

Keywords. stars:pre-main sequence, stars:mass loss, stars:magnetic fields, accretion disks

\section{Introduction}

I discuss in this contribution the accretion and ejection processes occurring in solartype young stars and their possible connection to the magnetic field. I will focus on the well studied $\mathrm{T}$ Tauri stars, pre-main sequence sources with typical mass $0.5 \mathrm{M}_{\odot}$, effective temperature $3500-4000 \mathrm{~K}$ (spectral types $\mathrm{K}-\mathrm{M}$ ) and radius $2 \mathrm{R}_{\odot}$. These young stars, with an age of a few million years, are still contracting towards the main sequence. A sub-class of T Tauri stars, the so-called Classical T Tauri stars or CTTs, are actively accreting matter from a circumstellar disk at a typical rate of $10^{-8} M_{\odot} y r^{-1}$ (see e.g. Ménard \& Bertout 1999 for a review) and are driving supersonic jets. Accretion and ejection signatures are strongly correlated in young stars (Cabrit et al. 1990, Hartigan et al. 1995). Understanding these two important processes, and their link, is one of the major issue in star formation. The magnetic field is thought to play a central role in both extracting the angular momentum from the disk and directing the accretion flow onto the star. I will focus in this contribution on two main critical, yet unsolved, issues: 1) the nature of the star-disk interaction, 2) the driving mechanism for the supersonic jets.

The exact nature of the star-disk interaction in T Tauri stars holds important clues on: the mechanism responsible for launching the jets, the regulation of stellar angular momentum as well as the physical parameters in the inner disc which control its evolution and the models of planetary formation. I will first describe in section $\S 2$ our current knowledge of surface magnetic fields in young stars, then review the arguments that have led to establish that dipolar stellar fields truncate the accretion disk and direct the accretion flow onto the star, in the so-called magnetospheric accretion scenario. I will 


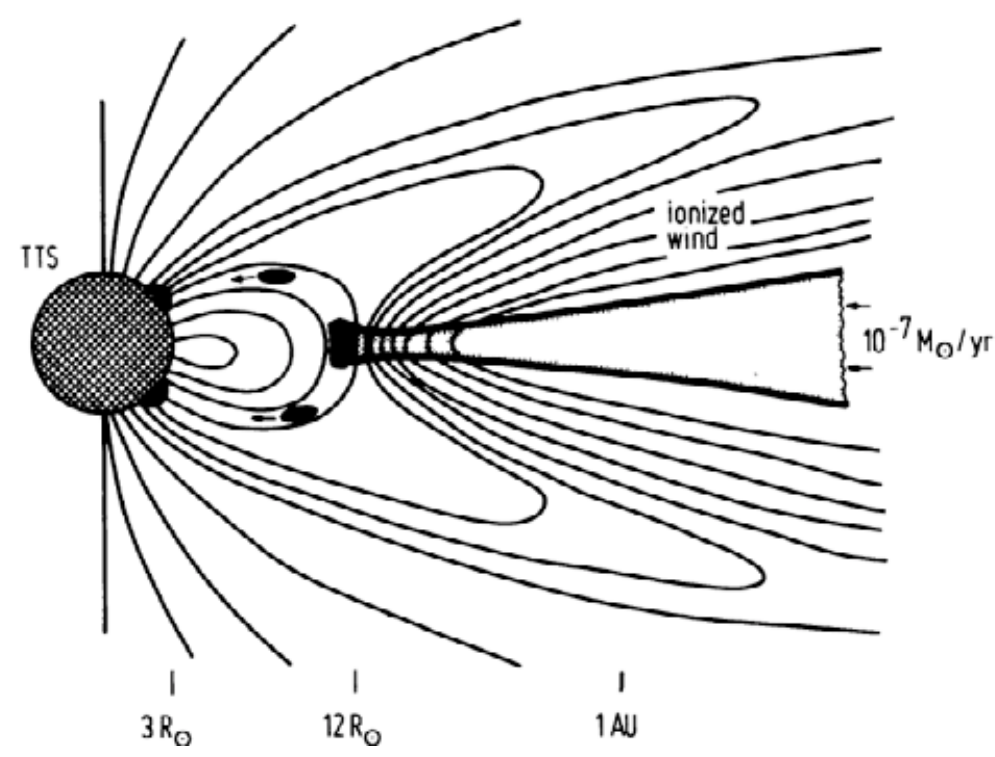

Figure 1. A sketch illustrating the basic concept of magnetospheric accretion in T Tauri stars (from Camenzind 1990).

then show results from recent monitoring studies that challenge the standard steady and axisymmetric picture of magnetically channeled accretion.

I turn in $\S 3$ to the origin of the supersonic atomic flows. I first describe their main observational characteristics, review the current magneto-hydrodynamic ejection models, both steady and non-steady, then present recent constraints obtained from high-angular resolution observations of the small scale jets from $\mathrm{T}$ Tauri stars. I also briefly discuss the origin of structures (knots) in jets and their relation to time variability. I finally summarize and discuss open questions in section $\S 4$.

\section{Accretion flow onto the star: the magnetospheric paradigm}

\subsection{Stellar magnetic field}

The first evidence for magnetic activity at the surface of young pre-main sequence stars came from their powerful X-ray and centimetric radio emissions (Montmerle 2002, André 1987). T Tauri stars show a strong correlation between $X$-ray and bolometric luminosities with an average $L_{X} / L_{b o l}$ ratio of $10^{-4}$, about 2 orders of magnitude higher than observed in the active Sun. X-ray flares, with properties similar to the ones observed in the Sun, are also detected in $\mathrm{T}$ Tauri stars. The light-curves show rapid rise in luminosity followed by an exponential decline over a characteristic time of $\simeq 10$ hours. The spectral properties of these flares indicate thermal bremsstrahlung emission from a hot cooling plasma with coronal electronic densities and temperatures. By analogy with the Sun, it is assumed that these flares arise in confined plasma loops. Using purely radiative cooling flare models and assuming equipartion field, loop sizes of a few stellar radii and surface magnetic fields on the order $\simeq 1 \mathrm{kG}$ are inferred. Indeed, mean field strengths of $2-3 \mathrm{kG}$ have been recently detected in a dozen $\mathrm{T}$ Tauri stars from Zeeman broadening measurements of photospheric lines (Guenther et al. 1999, Johns-Krull et al. 1999). This method does not provide information on the global geometry of the magnetic field. Circular polarization has been detected in the He I 5876 Åmission line (Johns-Krull et al. 1999), which is thought to form at the footpoint of the accretion funnel. Magnetic polarity therefore 
appears uniform in localized regions at the surface of TTs, suggestive of magnetic poles of a dipolar component. In the following, it will be assumed that such a component dominates at large distances from the star. In principle, the detailed geometry of the magnetic field can be reconstructed with Zeeman-Doppler imaging technics, which have been already extensively applied to the study of nearby cool stars (see the contribution by Moira Jardine, these proceedings, for more details). The high resolution spectropolarimeter ESPADONS being currently installed at the $\mathrm{CFH}$ telescope in Hawaii will soon allow to apply such mapping techniques to T Tauri stars.

\subsection{Magnetically channeled accretion}

The strong magnetic fields present at the surface of $\mathrm{T}$ Tauri stars are believed to significantly alter the accretion flow from the circumstellar disk to the stellar surface. Uchida (1983) and Uchida \& Shibata (1984) first suggested that CTTs magnetic fields disrupt the inner accretion disk, lifting material out of the disk plane towards the stellar magnetic pole. Camenzind (1990) and Kø̈nigl (1991) showed that the disk is expected to be truncated by the stellar magnetosphere at a few stellar radii, where the stellar magnetic field torque balances the viscous accretion disk torque, for typical mass accretion rates of $10^{-8} \mathrm{M}_{\odot} \mathrm{yr}^{-1}$ and dipolar magnetic fields of $1-3 \mathrm{kG}$. The basic concept of magnetospheric accretion is illustrated in Figure 1. Disk material is channeled from the disk inner edge to the stellar surface along magnetic field lines. The accretion flow then hits the stellar surface with close to free-falling velocities, giving rise to accretion shocks near the magnetic poles. The successes and limits of the current magnetospheric accretion models to account for the observational properties of CTTs are reviewed for example in Bouvier et al. (2004). Magnetospheric accretion models have been in particular successful at reproducing the shape and strength of the main hydrogen emission lines (Hartmann et al. 1994; Muzerolle et al. 2001), where all previous wind-based or chromospheric models had failed.

One of the main strength of the magnetospheric scenario is that it allows, in principle, to solve for the angular momentum problem in T Tauri stars. Young stars with accretion disks are observed to rotate slowly, at $\simeq 1 / 10$ of their break-up velocity, although they are expected to accrete large angular momentum material from the disk. For the magnetospheric accretion configuration to be stable on long timescales, the disk truncation radius must lie at or close to the co-rotation radius, ie the location in the disk where the keplerian velocity matches the equatorial velocity of the star (on the order of a few stellar radii in T Tauri stars). Stellar field lines threading the disk outside the co-rotation radius allow to transfer angular momentum outwards with a net braking effect on the star (Collier-Cameron \& Campbell 1993; Armitage \& Clarke 1996; Shu et al. 1994), providing the basis of what is referred to as the disk-locking mechanism.

\subsection{Challenges to the standard model}

\subsubsection{Departure from axisymmetry}

Young stars with active accretion disks show variations in luminosity that can be interpreted with bright $(\mathrm{T} \simeq 8000 \mathrm{~K}$ ) surface spots covering of order one percent of the stellar surface. These hot spots are identified as emission from shocked regions formed close to the magnetic poles at the footpoint of the accretion funnel (Bouvier \& Bertout 1989; Vrba et al. 1993). Rotational modulation is also detected in the fluxes and line-ofsight velocities of the main emission lines, formed in the hot plasma filling the accretion columns. These variabilities are interpreted as signatures of a magnetosphere tilted with respect to the rotation axis of the star (see e.g. Johns \& Basri 1995). 


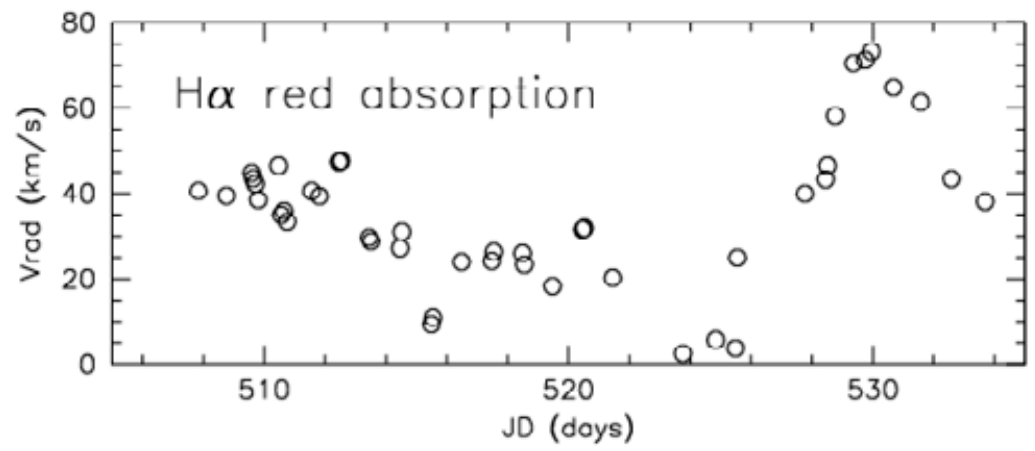

Figure 2. The observed variation over $\simeq 1$ month of the radial velocity of the redshifted absorption component in the $\mathrm{H}_{\alpha}$ line (from Bouvier et al. 2003). This variation is interpreted as a signature of magnetospheric inflation.

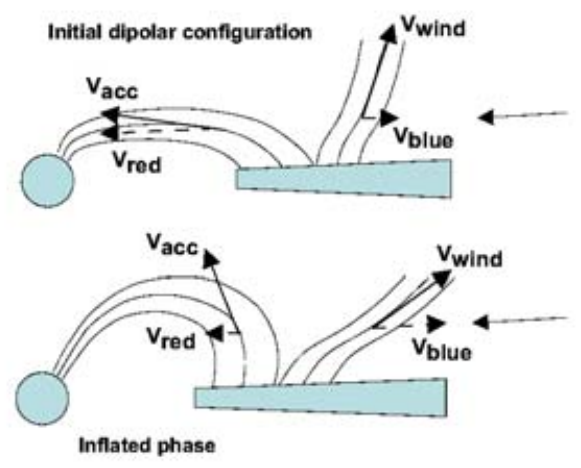

Figure 3. A sketch of the magnetospheric inflation scenario. The arrow on the right side indicates the line of sight to the AA Tau system (from Bouvier et al. 2003).

Further insight into the geometry of the star-disk interaction and its temporal evolution can be derived from spectro-photometric monitoring studies. The star AA Tau has been recently followed twice during $\simeq 4$ stellar rotation periods (Bouvier et al. 1999, 2003). The disk in this system is seen close to edge-on (inclination to the line-of-sight of $\mathrm{i} \simeq$ $75^{\circ}$ ). This peculiar geometry allows to study in details the critical region where the stellar magnetosphere interacts with the inner accretion disk. Recurrent eclipses of the photospheric light, with a period of 8.2 days, are reported in this star (Bouvier et al. 1999). These periodic occultations are interpreted as signatures of a non-axisymmetric warp of the inner disk located close to the co-rotation radius. The puffed inner disk rim periodically obscures the central star as it rotates at the keplerian rate. Such a warp of the inner disk is indeed expected to develop as a result of the interaction of the disk material with an inclined dipolar magnetosphere (Terquem \& Papaloizou 2000; Lai 1999; Romanova et al. 2003).

\subsubsection{A dynamical process?}

Magnetospheric accretion models assume that the stellar magnetosphere truncates the disk close to the co-rotation radius. However, the stellar magnetosphere interacts with the inner disk over a finite radial distance. The footpoints of the field lines anchored into the star and the ones anchored into the disk will therefore not rotate at the same rate. 
Recent numerical simulations indicate that such differential rotation induces a substantial distortion of the field lines over a timescale of a few keplerian periods at the inner disk. The response of the magnetic configuration to the differential rotation critically depends upon the magnitude of the magnetic diffusivity in the disk, a parameter which is still poorly constrained. If magnetic diffusivity is large, field lines can diffuse outwards in the disk, leading to magnetic flux expulsion (Bardou \& Heyvaerts 1996). In the opposite case, field lines are predicted to expand, open and eventually reconnect, leading to a restoration of the initial configuration (Hayashi et al. 1996, Goodson et al. 1997, Romanova et al. 2003). In such models, magnetospheric inflation cycles are expected to occur, accompanied by violent, episodic outflows and accretion events onto the star as the field lines open and reconnect.

A few observations seem to indicate that magnetospheric accretion in young stars is indeed a dynamical process. Time delays in the appearance of velocity components in the emission line profiles have been interpreted for possible evidence for magnetic field lines being twisted by differential rotation in SU Aur by Oliveira et al. (2000). Quasi-periodic X-ray flarings have been also reported for the embedded proto-stellar source YLW 15 (Tsuboi et al. 2000). They have been interpreted as recurrent reconnection events in a twisted magnetosphere. Our observations of the AA Tau system also indicate a strongly variable accretion process (Bouvier et al. 2003). In AA Tau the line-of-sight velocity of the redshifted absorption component in the $\mathrm{H}_{\text {alpha }}$ line is seen to slowly vary over typically 3 stellar rotation periods. The magnitude of this velocity component is thought to measure the curvature of the magnetospheric field lines which intersect the disk towards the observer (see Figure 3). In addition, a simultaneous blueshifted absorption feature is observed, strongly correlated in velocity with the redshifted absorption component. We interpret these velocity variations as signatures of magnetospheric inflation in AA Tau. As magnetic field lines expand due to differential rotation, the line-of-sight velocity of the accretion (resp. wind) flow decreases (resp. increases) due to projection effects. At the maximum inflation of the magnetosphere (ie when the radial velocity of the redshifted absorption component is minimum), all accretion signatures onto the star disappear, as would be expected due to the unfavorable configuration of field lines to launch disk material. Shortly thereafter, strong accretion resumes and the radial velocity of the redshifted component strongly increases, indicating that the magnetosphere has recovered its initial configuration. Obviously, monitoring campaigns on longer timescales would be required to fully confirm this scenario.

\section{Origin of the supersonic jets}

\subsection{Properties of the atomic jets}

At all stages where young stars are actively accreting matter (i.e for ages $\leqslant$ a few $10^{6}$ yrs), strong mass-loss signatures are also observed. The most spectacular ejection events occur during the earliest stages of star formation for ages $\leqslant 10^{5}$ yrs, when the central protostar is still embedded in its natal cocoon. Strongly collimated, supersonic $\left(\mathrm{v}_{\text {flow }}=\right.$ $300-400 \mathrm{~km} \mathrm{~s}^{-1}$ ) atomic flows, the so-called Herbig-Haro (hereafter $\mathrm{HH}$ ) jets, are detected out to distances of a few parsecs from their driving sources. These flows emit mostly in low excitation lines of weakly ionized atoms (the most prominent being [O I] $6300 \AA$, [S II] $6716,6731 \AA$ and [N II] $6584 \AA$ ), characteristic of excitation temperatures in the range of $8000-10^{4} \mathrm{~K}$. At later ages $\left(\geqslant 10^{6} \mathrm{yrs}\right)$, when the surrounding envelope dissipates and the central source becomes an optically visible $\mathrm{T}$ Tauri star, both accretion and ejection signatures strongly decrease in strength but their correlation persists. With the 


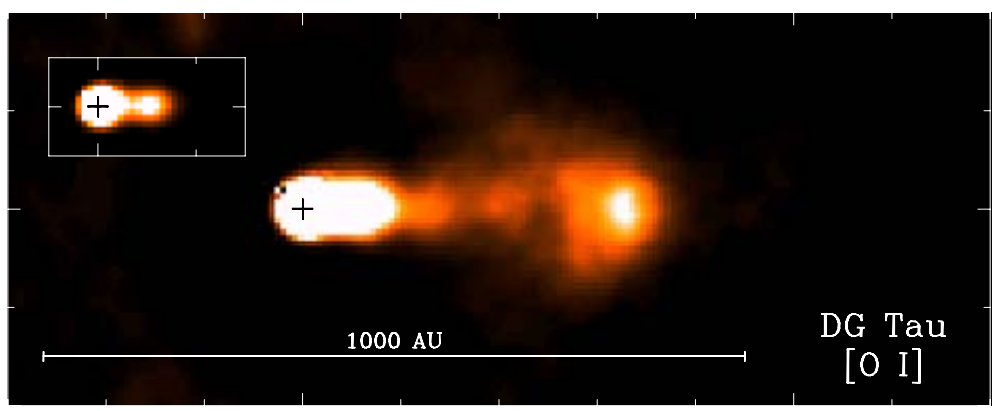

Figure 4. Map in the [O I] $6300 \AA$ line of the small scale jet around the T Tauri star DG Tau. The cross locates the centroid of the continuum emission (star position). The insert at the upper left corner shows the central arc-second of the jet emission. Adapted from Dougados et al. (2000).

recent advance of high-angular resolution technics, small scale jets extending out to a few hundred AUs have been clearly identified in the vicinity of T Tauri stars (see Figure 4). These microjets are the smaller scale version of the more massive $\mathrm{HH}$ jets emerging from younger sources, clear confirmation that the same physical mechanism is at play through all phases of star formation and that it is intimately connected with the accretion process. Although less powerful, these small scale jets are precious in that they give access to the innermost regions of the flow (central $100 \mathrm{AU}$ ) where models predict that most of the collimation and acceleration processes occur.

\subsection{Magneto-centrifugal ejection}

The physical mechanism by which mass is ejected from young stars and collimated into jets remains a fundamental open issue in star formation theory. The strong correlation between ejection and accretion found in PMS stars, with a mass flux ratio as high as 0.1 (Cabrit et al. 1990; Hartigan et al. 1995) has favored accretion-driven magnetohydrodynamic (MHD) wind models. A large scale magnetic field, either originating in the disk or in the star, provides a mechanism to extract angular momentum from the star-disc system and collimate the flow on small scales. However, it is not yet established whether the jet originates from the stellar surface, the magnetosphere/disk interface, or a wide range in disk radii; whether it is launched mostly by magneto-centrifugal forces or by a strong thermal pressure gradient in an accretion-heated corona; and whether it is a steady process or not. Answering these questions is crucial not only for the jet phenomenon in itself, but also for models of exoplanet formation, as they have distinct implications on the internal structure, angular momentum transfer, and heating/irradiation processes in the inner regions of protoplanetary disks.

\subsection{Steady wind models}

Three classes of stationary magnetic wind models have been developed: pure stellar winds (e.g. Sauty \& Tsinganos 1994), extended disk winds (Blandford \& Payne 1982; Ferreira 1997) and winds originating from the star-disk interaction (X-wind: Shu et al. 1995, reconnection X-winds: Ferreira et al. 2000). These models are distinguished by whether they are powered by accretion (disk winds and X-winds) or by the protostellar rotational energy (stellar winds, Reconnection X-winds) and whether the disk is threaded by a significant magnetic field or not (extended disc winds vs $\mathrm{X}$-wind and stellar winds). In the case of the stellar and disk winds, self-similar solutions have been found. In the X-wind solution of F. Shu \& collaborators, mass-loss originates from a single point in the disk (the inner disk radius), which leads to an intrinsically non self-similar behavior. Self-similar 
steady disk wind solutions require a large-scale dipolar magnetic field threading the disk and that the magnetic torque dominates over the viscous torque. Recent numerical simulations have confirmed that indeed, under such conditions, steady disk ejection can occur (Casse \& ferreira 2004).

Observational predictions have been recently computed for steady disk and X-wind models (Garcia et al. 2001, Shang et al. 2002), which can be confronted to observations of the inner regions of jets from $\mathrm{T}$ Tauri stars. Both these models quantitatively reproduce some general global properties of jets such as: ejection to accretion rates, terminal velocities, collimation scales and jet widths. The most stringent constraint to date on the jet launching radius comes from the detection of rotation signatures in a few $\mathrm{T}$ Tauri jets (Bacciotti et al. 2002; Coffey et al. 2004). The ratio of poloidal velocity to specific angular momentum $\left(\mathrm{r} \times \mathrm{v}_{\phi}\right)$ measured in the jet, at a given altitude above the disk, provides a model-independent measure of the launching radius (Bacciotti et al. 2002; Anderson et al. 2003). Observations suggest launching radii in the range 0.1-3 AU, which indicate that a substantial disk wind component is present at the $\mathrm{T}$ Tauri phase. Observational predictions strongly depends on the assumed heating mechanism. The properties of the line emission from these jets requires that temperatures on the order of $8000 \mathrm{~K}$ be sustained on scales $\geqslant 1000$ au. This is still an unsolved problem. Detailed studies of line ratios in 2 jet systems have shown that shock heating best reproduce the observations (Lavalley-Fouquet et al. 2000, Dougados et al. 2000). Time variability may therefore play an important role in structuring the jets. We will come back to this issue later.

\subsection{Non-steady ejection models}

Another class of models have considered the possibility that jets in young stars originate from intrinsically non-steady processes. In the cyclic magnetospheric inflation scenario of Hayashi et al. (1996) and Goodson et al. (1997), episodic outflows occur as a consequence of reconnection events in the magnetosphere. These CME-like ejections could in particular account for some of the variable outflow signatures observed in young stars. Indeed, strong variability in the high-velocity wings of the line emission profiles are observed in young stars (Solf 1994). An expanding shocked bubble has also been reported in the case of XZ Tau (Coffey et al. 2004). These episodic ejection events will however require an external pressure, in excess of the one provided by the interstellar medium, to collimate them.

Time dependent numerical simulations of wind generation from the disk have been performed by numerous authors. In these simulations, the disk is usually treated as a boundary condition (Ustyugova et al. 1999, 2000; Ouyed \& Pudritz 1997, 1999; Krasnopolsky et al. 1999). Simulations taking into account the accretion flow, but still in the ideal MHD case, have been computed by Uchida \& Shibata (1985), Shibata \& Uchida (1986, 1990), Stone \& Norman (1994), Kudoh et al. (1998), Kato et al. (2002). However, realistic computations with both the accretion flow and diffusivity effects in the disk are still scarce. Kuwabara et al. (2000) investigated the effect of magnetic diffusivity in the disk and found that when this parameter is low, mass accretion and jet formation takes place intermittently.

\subsection{Origin of jet structures}

One important morphological property of jets is the fact that the emission is dominated by knots, usually spatially compact close to the central source, but resolved into extended bow-shaped structures at larger distances. Several competing models have been proposed to explain the ubiquitous presence of knots in stellar jets: (1) variability in the jet ejection velocity and/or direction (Raga \& Kofman 1992), (2) Kelvin-Helmholtz instabilities at 
the jet/cloud interface (e.g. Micono et al. 2000), (3) internal MHD or current-driven instabilities developing in the jet (e.g. Appl et al. 2000; Baty \& Keppens 2002). Knot kinematical properties, in particular proper motions, seem to favor the first alternative. Very different variability timescales are involved: from $\Delta T \simeq 10^{4}$ yrs, corresponding to the bow-shocks observed on distances of typically $0.1 \mathrm{pc}$ in $\mathrm{HH}$ jets from embedded sources, to $\Delta T \simeq 1-10$ yrs for the small scale knots located at the base $(\mathrm{d}<1000 \mathrm{AU})$ of $\mathrm{T}$ Tauri jets. The extended bow-shocks seem related to the strong thermal instability events massive disks undergo during the earliest stages of their evolution, leading to powerful outbursts in luminosity of the central system (the so-called Fu Ori events). A significant fraction of the mass of the central protostar could be accreted during this stage. Small scale knots prevalent at the wind base, on the other hand, correspond to moderate shock velocities $\left(\leqslant 50-70 \mathrm{~km} \mathrm{~s}^{-1}\right)$, i.e. moderate perturbations of the underlying average flow. One possible origin for the short timescale variations could be instabilities at the interface region between the stellar magnetosphere and the disk leading to episodic mass ejection events, such as the cyclic inflation scenario presented earlier. Numerical simulations currently fail however to reproduce the typical 1-10 yrs timescales (cf e.g. Matt et al. 2002): the natural timescale coming out of these simulations correspond to the orbital period at the inner disk edge $\simeq 1$ week. One alternative possible could be that the short timescale variations are linked to a magnetic cycle similar to the one observed in the Sun, with recurrent polarity reversals induced by the stellar dynamo. We note that although the origin of these small-scale perturbations is still unknown, they should not significantly affect the conclusions reached by comparing the average flow properties with a stationary wind model.

\section{Summary and Open questions}

There is now strong observational evidence that the accretion flow in $\mathrm{T}$ Tauri stars is channeled by the stellar magnetosphere and that the star-disk interaction is a nonaxisymmetric and time dependent process. Important questions are still raised: 1) what is the geometry of the stellar magnetosphere? Does the dipolar component dominate at the distance of $\simeq 0.1 \mathrm{au}$, where interaction with the inner accretion disk is predicted to occur? We can hope to get some answers to this question in the near-future with the application of Zeeman-Doppler imaging technics to the study of T Tauri stars. Another theoretical question that is raised is: can the magnetosphere sustain the accretion flow at the rates deduced from the observations and how? What is the stability of this structure and how is the helicity evacuated? In particular do reconnection events and episodic outflows occur in young stars as a consequence of differential rotation ? Long-term spectro-photometric monitoring studies of a large sample of $\mathrm{T}$ Tauri stars are required to tackle this issue.

Large scale magnetic fields seem required to launch and collimate the supersonic atomic jets. Recent detection of rotation rates in jets indicate that an extended disk wind component is present at the $\mathrm{T}$ Tauri phase. This implies that the inner disk (out to $\simeq 3 \mathrm{AUs}$ typically) is magnetized, which may have strong implications for planetary formation and migration models (Terquem 2003). The origin of this large scale magnetic field, whether advected from the interstellar medium or dynamo, is still an open question. The existence of an additional (unsteady?) wind component originating from the star-disk interface is also still under debate. A related unsolved issue is the origin of the outflow variability observed on short timescales (1-10 yrs) and its connection to magnetic processes. Finally, how does the transition occur between these massive young winds and the solar-type winds observed on the main sequence? 


\section{Acknowledgements}

I would like to thank the organizers for this very pleasant and instructive meeting, that allowed me to get introduced to the world of coronal mass ejections as well as get a glimpse of Beijing. I warmly thank them for their hospitality and the financial support provided.

\section{References}

Anderson, J.M., Li, Z.-Y., Krasnopolsky, R., \& Blandford, R.D. 2003 ApJ 590, L107

André, P. 1987, in: Protostars and Molecular Clouds, eds. T. Montmerle \& C. Bertout, p. 143

Appl, S., Lery, T., \& Baty, H. 2000, A\&A, 355, 818

Armitage, P.J. \& Clarke, C.J. 1996, MNRAS, 280, 458

Bacciotti, F., Ray, T., Mundt, R., Eislöffel, J., \& Solf, J. 2002, ApJ 576, 222

Bardou, A. \& Heyvaerts, J. 1996, A\&A, 307, 1009

Baty, H. \& Keppens, R. 2002, ApJ 580, 800

Bouvier, J. \& Bertout, C. 1989, A\&A, 211, 99

Blandford, R.D. \& Payne D.G. 1982, MNRAS 199, 883

Bouvier, J., Chelli, A., Allain, S., et al. 1999, A\&A, 349, 619

Bouvier, J., Grankin, K., Alencar, S., et al. 2003, A\&A, 409, 16

Bouvier, J., Dougados, C., \& Alencar, S. 2004 Ap\&SS, 292, 6599

Cabrit, S., Edwards, S., Strom, S.E., \& Strom, K.M. 1990, ApJ, 354, 687

Camenzind, M. 1990, Reviews of Modern Astronomy, 3, 234

Coffey, D., Bacciotti, F., Woitas, J., Ray, T.P., \& Eislffel, J. 2004, ApJ 604, 758

Collier Cameron, A. \& Campbell, C.G. 1993, A\&A, 274, 309

Casse, F. \& Ferreira, J. 2004, ApJ 601, L139

Dougados, C., Cabrit, S., Lavalley, C., \& Ménard, F. 2000, A\&A 357, L61

Ferreira, J. 1997, A\&A 319, 340

Ferreira, J., Pelletier, G., \& Appl, S. 2000, MNRAS 312, 387

Garcia, P.J.V., Cabrit, S., Ferreira, J., \& Binette, L. 2001, A\&A 377, 609

Goodson, A.P., Winglee, R.M., \& Boehm, K. 1997, ApJ, 489, 199

Guenther, E.W., Lehmann, H., Emerson, J.P., \& Staude, J. 1999, A\&A, 341, 768

Hartigan, P., Edwards, S., \& Ghandour, L. 1995, ApJ, 452, 736

Hartmann, L., Hewett, R., \& Calvet, N. 1994, ApJ, 426, 669

Hayashi, M.R., Shibata, K., \& Matsumoto, R. 1996, ApJ, 468, L37

Johns, C.M. \& Basri, G. 1995a, ApJ, 449, 341

Johns-Krull, C.M., Valenti, J.A., \& Koresko, C. 1999, ApJ, 516, 900

Kato, S.X., Kudoh, T., \& Shibata, K. 2002 ApJ, 565, 1035

Kø̈nigl, A. 1991, ApJ, 370, L39

Krasnopolsky R., Li, Z.Y., \& Blandford, R. 1999, ApJ 526, 631

Kudoh, T., Matsumoto, R., \& Shibata, K. 1998, ApJ 508, 186

Kuwabara, T., Shibata, K., Kudoh, T., \& Matsumoto, R. 2000, PASJ 52, 1109

Lavalley-Fouquet, C., Cabrit, S., \& Dougados, C. 2000, A\&A 356, L41

Matt, S., Goodson, A.P., Winglee, R.M., et al. 2002, Ap\&J, 574, 232

Ménard, F. \& Bertout, C. 1999, NATO ASIC Proc. 540: The Origin of Stars and Planetary Systems, 341

Micono, M., Bodo, G., Massaglia, S., Rossi, P., Ferrari, S., \& Rosner, R. 2000, A\&A 360, 795

Montmerle, T. 2002, EAS Publications Series, Volume 3, Proceedings of Star Formation and the Physics of Young Stars, held 18-22 September, 2000 in Aussois France. Edited by J. Bouvier and J.-P. Zahn. EDP Sciences, p 85.

Muzerolle, J., Hartmann, L., \& Calvet, N. 2001, ApJ, 550, 944

Oliveira, J.M., Foing, B.H., van Loon, J.T., \& Unruh, Y.C. 2000, A\&A, 362, 615

Ouyed, R. \& Pudritz, R.E. 1997, ApJ 484, 794

Ouyed, R. \& Pudritz, R.E. 1999, MNRAS 309, 233

Raga, A. \& Kofman, L. 1992, ApJ 386, 222 
Sauty, C. \& Tsinganos, K. 1994, A\&A 287, 893

Shang, H., Glassgold, A.E., Shu, F.H., \& Lizano, S. 2002, ApJ 564, 853

Shibata, K. \& Uchida, Y. 1986, PASJ 38, 631

Shibata, K. \& Uchida, Y. 1990, PASJ 42, 39

Stone, J.M. \& Norman, M.L. 1994 ApJ 433, 746

Shu, F., Najita, J., Ostriker, E., Wilkin, F., Ruden, S., \& Lizano, S. 1994, ApJ, 429, 781

Shu, F.H., Najita, J., Ostriker, E.C., \& Shang, H. 1995, ApJ 455, L155

Solf, J. 1994 in ASP Conf. Ser., Vol 57, Stellar and Circumstellar Astrophysics, eds. G. Wallerstein \& A. Noriega-Crespo, p 22

Terquem, C. 2003, MNRAS 341, 1157

Terquem, C. \& Papaloizou, J.C.B. 2000, A\&A, 360, 1031

Tsuboi, Y., Imanishi, K., Koyama, K., et al. 2000, ApJ 532, 1089

Romanova, M.M., Ustyugova, G.V., Koldoba, A.V., Wick J.W., \& Lovelace, R.V.E. 2003, ApJ, 595, 1009

Uchida, Y. 1983, in Activity in Red-Dwarf Stars, Reidel, Dordrecht, P.B Byrne \& M. Rodono Eds., p. 625.

Uchida, Y. \& Shibata, K. 1984, PASJ 36, 105

Uchida, Y. \& Shibata, K. 1985, PASJ 37, 515

Ustyugova, G.V., Koldoba, A.V., Romanova, M.M., Chechetkin, V.M., \& Lovelace, R.V.E. 1999, ApJ 516, 221

Ustyugova G.V., Lovelace, R.V.E., Romanova, M.M., Li, H., \& Colgate, S.A. 2000, ApJ 541, L21

Vrba, F.J., Chugainov, P.F., Weaver, W.B., \& Stauffer, J.S. 1993, AJ, 106, 1608

\section{Discussion}

ShiBATA: - You mentioned that the first resistive MHD simulations of jets from a disk has been done by Casse, but this is not correct. Actually, Kawabara et al. (2002 PASJ, $\mathrm{v} 52,1109)$ was the first. In relation to this, you mentioned that the steady solution was obtained by a resistive model, while the ideal MHD model does not lead to steady state if a disk is included, and gave an impression that the resistive steady model is better. But we should remember that a resistive steady model is very idealized and probably very unrealistic. This is because magnetorotational instability occurs in the disk to produce time variability, turbulence, and effective viscosity. Actual disk and associated ejection of jets would be very dynamic and time dependent like solar corona. You showed interesting $\mathrm{H} \alpha$ observations of star-disk interaction by Bouvier et al. (2003). Does it show only accretion? Is there a possibility that mass ejections are associated?

Dougados: The radial velocity component I discussed is associated to the accretion process. However there is also a blueshifted radial velocity component anti-correlated with the redshifted radial velocity and which we believe traces the base of the wind. See Bouvier et al. (2003) for more details. 